\title{
A new MIMO system using Modified Hermite Pulses Compared to MIMO-OFDM system
}

\author{
D.Naar ${ }^{1}$, M.Bouziani ${ }^{2}$ \\ ${ }^{1}$ (Member in Laboratory: Telecommunications and Digital Signal Processing Djillali Liabes University of \\ SidiBel Abbes .Algeria), ${ }^{2}$ (Member in TDSP DLU Sidi Bel Abbes .Algeria)
}

\begin{abstract}
This paper is intended to compare between a new MIMO system proposed using the Modified Hermite pulses and MIMO-OFDM system in the coherent case (the channel is supposed perfectly known by the receiver). The studied system exploit space time block coding and integrate the technique BICM as a powerful tool to ensure a robust communication. The estimate of the symbols in reception is articulated on the maximum likelihood criterion. Simulations results proved that the MIMO system using modified Hermite pulses offers better performances than the MIMO-OFDM system.
\end{abstract}

Keywords: - MIMO, Hermite pulses, STBC, OFDM, BICM.

\section{INTRODUCTION}

The Hermite functions constitute an orthogonal waveforms. To be used as support of transmission, the operation requires that they must be normalized. At the receiver, the orthogonality of these functions guarantees the capability to recover the original data transmitted. The Hermite pulses and their applications were introduced in several papers. A new N-dimensional digital modulation technique based on the use of orthogonal waveforms derived from Hermite polynomials is studied in [3]. A novel modified Hermite polynomial functions for use in impulse radio (Ultra Wideband) communication is proposed in [4] The work of [5] shows the effectiveness of the orthogonality of Hermite waveforms to increase data rate and to add error correction code to achieve reliable communication in a UWB system. The UWB system received much interest in recent years, thanks to these proprieties of use for short distance for communications in a dense multipath environment [6]. Another application of the Hermite waveform is that inroduced in [7]. There, Hermite wavelets are used to replace sinusoid waves in multicarrier system in a high-rate digital subscriber loop (DSL). Our objective in this paper, is to exploit the orthogonal Hermite pulses in a system of transmission MIMO, and to compare its performances with its Counterpart MIMO OFDM.

To make the system reliable and optimal, the space-time coding is used if the channel is known perfectly by the receiver (coherent case). The system MIMO suggested in this article has two transmitting antennas and two reception antennas. To ensure a robust communication, the solution is to use the technique known as bit interleaved coded modulation (BICM). The organization of this work is as follows. In Section II, we describe the system overview of the MIMO system based on orthogonal Hermite Pulses. In this Section, we describe the receiving system. In Section III, we show the simulation results for the proposed system and those of system MIMO_OFDM. Finally, section IV concludes the paper.

\section{SYSTEM OVERVIEWS}

\section{A-Channel Model}

The idea of MIMO systems is to combine the spatial diversity at transmitter to the spatial diversity reception. This variety ensures safer communication and avoids the inconvenience associated with propagation conditions such as multipath and attenuation. Moreover, the probability of losing any information decreases exponentially with the number of uncorrelated antennas [1].

We use this product in a single-user model in a non-selective channel in frequency as limited to a narrow band. This model consists of a matrix $\mathbf{H}$, each coefficient $\boldsymbol{h}_{j i}$ represents the complex transfer function that characterizes the path between the $i^{\text {th }}$ transmitting antenna and the $j^{\text {th }}$ receiving antenna. At the reception, each antenna receives the sum of symbols emitted simultaneously by each of the $N_{t}$ antennas. The signal received by the $j^{\text {th }}$ antenna can be written:

$y_{j}=\sum_{i=1}^{N t} h_{j i} s_{i}+b_{j}$ 
Where $b_{j}$ is a sample of Additive White Gaussian Noise (AWGN).

For $N_{r}$ receiving antennas, the received vector $\mathbf{y}$ can be written in the following matrix from:

$\mathbf{y}=\mathbf{H s}+\mathbf{b}$

With $\mathbf{S}=\left[s_{1} \ldots \ldots s_{N t}\right]^{T}$ the vector of symbols transmitted, $\mathbf{H}$ the channel matrix of $N_{r} \times N_{t}$ And $\mathbf{b}=\left[b_{1} \ldots \ldots b_{N r}\right]^{T}$ the vector of Additive White Gaussian Noise. We assume that $E\left[s s^{*}\right]=\mathbf{I}_{N t}, E\left[b b^{*}\right]=\mathbf{R}$ (the correlation matrix of noise) and $E\left[s b^{*}\right]=0$.

Several works are to assume that the elements of $\mathbf{H}$ are uniformly distributed phase and amplitude follows a Rayleigh law. This model is typical of an environment with many echoes and a sufficient gap between the antennas. We also assume that the channel remains constant during transmission of a data block and the receiver knows the channel matrix $\mathbf{H}$. This knowledge can be obtained either by symbols of learning is by blind channel estimation. Figure 1 shows the MIMO model.

\section{B - Modified Hermite Pulses}

It is clear that The Hermite orthogonal functions are not finite time functions. To be used as communication symbols, the Hermite functions need to be scaled to fit into a designated symbol period [3].

In this work, the Hermite Functions are scaled and adapted to the digital transmission according to a numerical method listed in [3]. it is supposed that the Hermite pulses contain almost the same quantity of energy which is equal to the unit.

\section{C- Transmission System}

The BICM-MIMO using Hermite pulses is depicted in Fig. 1. The information bits are first encoded by a rate-r convolutional encoder. The coded bits are then interleaved by a random permutation. The interleaved coded bits are mapped to complex symbols in the considered phase constellation. After the mapping, the symbols are carried on a corresponding Hermite Wiveforms. The complex symbols is then split into two subblocks, corresponding to the two transmit antennas. The signals to be transmitted to the two antennas in one symbol period are described as follows:

$$
\begin{aligned}
& s_{1}(t)=\sum_{i=0}^{N / 2-1} c_{i} g_{i}(t) \\
& s_{2}(t)=\sum_{i=N / 2}^{N-1} c_{i} g_{i}(t)
\end{aligned}
$$

Where $c_{i}$ is the $i^{\text {th }}$ complex symbol (or real symbol) and $g_{i}(t)$ is corresponding basis pulse.

The signals $s_{1}$ and $s_{2}$ are organized in space and time according to Alamouti space time coding by forming the following code matrix.

$$
\mathbf{S}=\left[\begin{array}{cc}
S_{1} & -s_{2}^{*} \\
S_{2} & S_{1}^{*}
\end{array}\right]
$$

The rows 1 and 2 of the matrix correspond to the signals transmitted from antennas 1 and 2 respectively. The columns correspond to the first symbol period and second symbol period.

The received signals, after passing through the channel for two symbols periods are expressed by :

$$
\left[\begin{array}{ll}
y_{11} & y_{12} \\
y_{21} & y_{22}
\end{array}\right]=\left[\begin{array}{ll}
h_{11} & h_{12} \\
h_{21} & h_{22}
\end{array}\right]\left[\begin{array}{cc}
s_{1} & -s_{2}^{*} \\
s_{2} & s_{1}^{*}
\end{array}\right]+\left[\begin{array}{ll}
b_{11} & b_{12} \\
b_{21} & b_{22}
\end{array}\right]
$$


After space time decoding, the data carried on the $p^{\text {th }}$ basis orthogonal Hermite pulses can be recovered by using a correlator detector "integrate and dump".

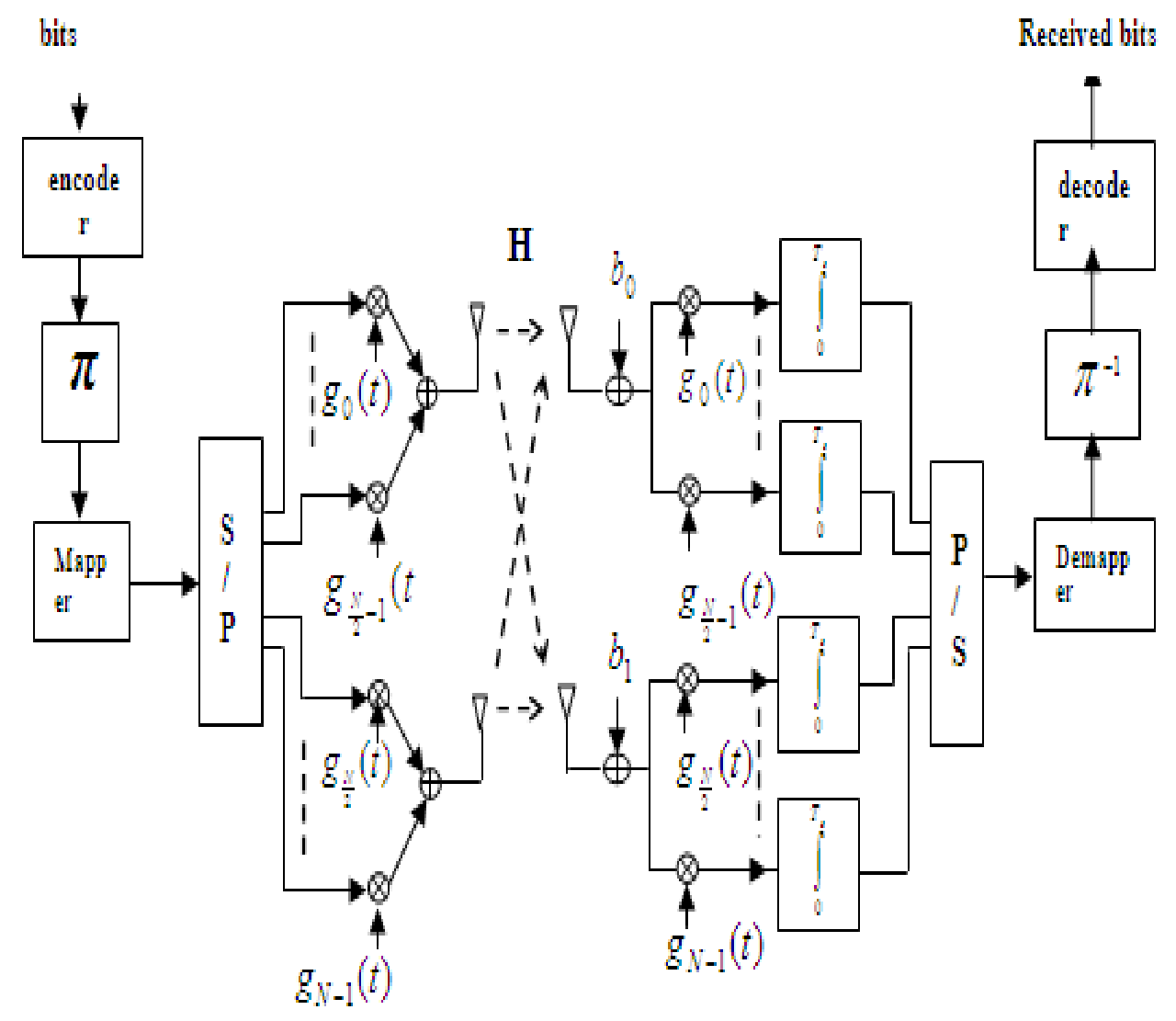

Fig, 1 : A Block Diagram of the MIMO system Based on Modified Hermite Pulses.

Let $s_{1}(t)$ is signal received by antenna 1 . Assuming that synchronization is perfect, the signal $s_{1}(t)$ Is multiplied by $g_{p}(t)$. Then the product is integrated over the symbol period. The implementation can be expressed as:

$\int_{0}^{T_{s}} s_{1}(t) g_{p}(t) \cdot d t=\int_{0}^{T_{s}}\left[g_{p}(t) \sum_{i=0}^{\frac{N}{2}-1} c_{i} g_{i}(t)\right] \cdot d t=\sum_{i=0}^{\frac{N}{2}-1} c_{i} \int_{0}^{T_{s}} g_{p}(t) \cdot g_{i}(t) \cdot d t=c_{p} \delta_{p p} \cdot(7)$

Where $\delta_{p p}$ is a positive constant.

III- A: Simulation Parameters

\section{SIMULATION RESULTS}

In this section, we analyze the performance of MIMO-OFDM system without guard interval and the proposed system which is based on modified Hermite pulses.

Simulations are conducted in Rayleigh fading channel. It is assumed that perfect CSI is known at the receiver.

\section{III-B: Performance Comparison}

Fig. 2 and 3 show uncoded BER performances of MIMO-OFDM system and MIMO based on Modified Hermite pulses with $2 \times 2$ antennas in i.i.d. fading channels. As shown in Fig.1, the simulation is carried out BPSK and QPSK modulation technique with using Alamouti space time coding. The receiver use a hard decision to decode the transmitted bits.

Fig.4 and 5 show the BER performance comparison of MIMO_-OFDM and The MIMO based on Modified Hermite Pulses when the BICM (Bit Interleaved Coded Modulation) is used with [5 7] encoder. 


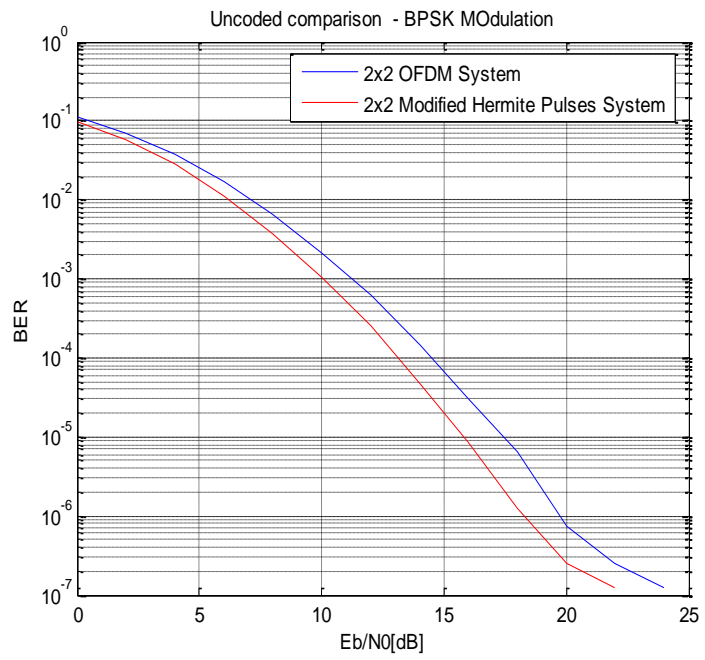

Fig.2: The uncoded BER Performance comparison of MIMO-OFDM system and MIMO Using Modified Hermite Pulses (MPH) for a BPSK modulation

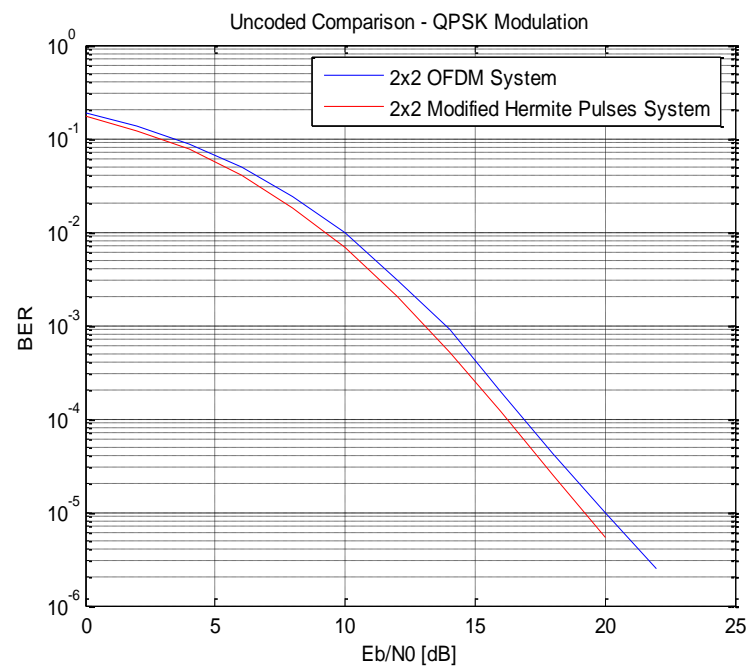

Fig.3: The uncoded BER Performance comparison of MIMO-OFDM system and MIMO Using Modified Hermite Pulses (MPH) for a QPSK modulation

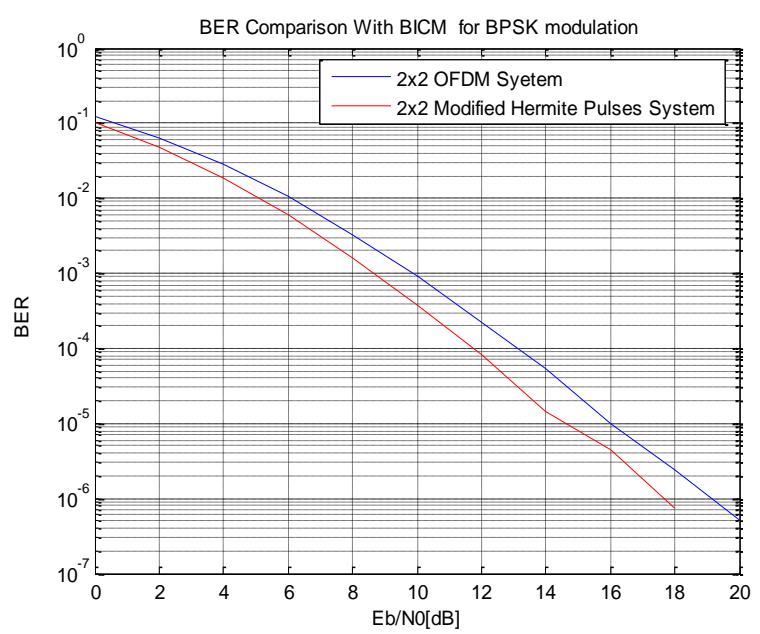

Fig.4: The coded BER Performance comparison of MIMO-OFDM system and MIMO Using Modified Hermite Pulses (MPH) for a BPSK modulation with [5 7] encoder 


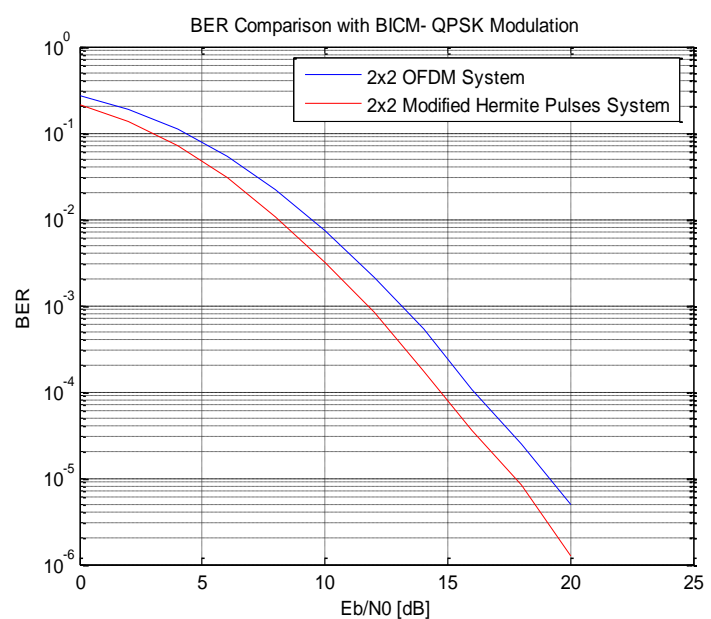

Fig.5: The coded BER Performance comparison of MIMO-OFDM system and MIMO Using Modified Hermite

Pulses for a QPSK modulation with [5 7] encoder

\section{CONCLUSION}

In this paper, the performance of the MIMO system using Hermite orthogonal functions compared to the MIMO- OFDM system is studied. In this context, we combined with BICM technique. As a result, MIMO system based on Hermite pulses provides a very high performance, and easy to decode system for broadband wireless communications.

\section{REFERENCES}

[1]. Alamouti S., "A simple transmit diversity technique for wireless communications," IEEE J. Select. Areas Comm., vol. 16, pp. 1451-1458, Oct. 1998.

[2]. G. Caire, G. Taricco, E. Biglieri, "Bit-interleaved coded modulation," IEEE Trans. Inform. Theory, vol. 44, pp.927-946, May1998.

[3]. W. Chongbure, «Digital Transmission by Hermite N-dimensional Antipodal scheme». Blacksburg, Virginia, pages 9-19. February11, 2004

[4]. M.Ghavami, L.B.Michael, S.Haruyama, and R.Kohno, "A novel UWB pulse shape modulation system ," Kluwer International Journal on Wireless Personal Communications, vol. 23, no.1,pp.105-120,2002.

[5]. Mitchell, C. and Kohno, R., "High Data Rate Transmissions Using Orthogonal Modified Hermite Pulses in UWB Communications," International Conferences on Telecommunications, ICT 2003, vol. 2, pp. 1278-1283.

[6]. Win, M. Z. and Scholtz, R. A., "Impulse Radio: How It Works," IEEE Communications Letter, vol. 2, no. 2, pp. 36-38, February, 1998.

[7]. Martens, J. B., "The Hermite Transform Theory," IEEE Trans. Acoustics, Speech, and Signal Processing, vol.38, no.9, pp. 1595-1606, September 1990. 